\title{
Modeling the irradiance dependency of the quantum efficiency of photosynthesis
}

\author{
Greg M. Silsbe and Jacco C. Kromkamp \\ Royal Netherlands Institute for Sea Research (NIOZ-YE). Postbus 140, 4400 AC. Yerseke, Netherlands
}

\begin{abstract}
Measures of the quantum efficiency of photosynthesis $\left(\phi_{\text {PSII }}\right)$ across an irradiance (E) gradient are an increasingly common physiological assay and alternative to traditional photosynthetic-irradiance (PE) assays. Routinely, the analysis and interpretation of these data are analogous to PE measurements. Relative electron transport rates $\left(\mathrm{rETR}=\mathrm{E} \times \phi_{\mathrm{PSI}}\right)$ are computed and fit to a PE curve to retrieve physiologically meaningful $\mathrm{PE}$ parameters. This widespread approach is statistically flawed as the response variable ( $\mathrm{EETR}$ ) is explicitly dependent on the predictor variable (E). Alternatively the E-dependency of $\phi_{\text {PSII }}$ can be modeled directly while retaining the desired PE parameters by normalizing a given PE model to E. This manuscript presents a robust analysis in support of this alternative procedure. First, we demonstrate that scaling $\phi_{\text {PSII }}$ to rETR unnecessarily amplifies the measurement error of $\phi_{\mathrm{PSII}}$ and using a Monte-Carlo analysis on synthetic data induces significantly higher uncertainty in computed PE parameters relative to modeling the E-dependency of $\phi_{\mathrm{PSI}}$ directly. Next a large dataset is simultaneously fitted to four PE models implemented in their original and E-normalized forms. Four statistical criteria used to evaluate the efficacy of nonlinear models demonstrate improved model fits and more precise PE parameters when data are modeled as E-dependent changes in $\phi_{\text {PSII }}$. The analysis presented in this manuscript clearly demonstrates that modeling the E-dependency of $\phi_{\text {PSII }}$ directly should be the norm for interpreting active fluorescence measures.
\end{abstract}

Active fluorescence measures across a defined actinic irradiance (E) gradient can be used to examine the E-dependency of quantum efficiency of photosynthesis $\left(\phi_{\text {PSII }}\right)$, photochemical quenching $\left(\mathrm{q}_{\mathrm{p}}\right)$, nonphotochemical quenching (NPQ), and relative electron transport rates (rETR). Although such measurements are often referred to as rapid light curves (White and Critchley 1999), here we adopt the term fluorescence light curve (FLC) to reflect that most commercially available fluorometers permit the user to define the magnitude and duration of the E gradient. FLCs are simple to perform, nondestructive, can be made in high frequency, and provide data immediately (i.e., do not require lengthy incubations). As such, they are becoming an increasingly popular alternative to traditional in vitro photosynthetic-irradiance (PE) assays (Sug-

${ }^{\star}$ Corresponding author: E-mail: greg.silsbe@nioz.nl

\section{Acknowledgments}

This research has been made possible by EU-FP7 grant PROTOOL (contract number 226880) and the NWO-ALW-ZKO grant IN PLACE. We like to thank the IN PLACE team, and Katja Philippart in particular, for providing the nutrient data and logistic support. Comments by an anonymous reviewer improved this manuscript.

DOI 10.4319/lom.2012.10.645 gett et al. 2011). While the statistical treatment of in vitro PE assay data have been examined (Frenette et al. 1993; Aalderink and Jovin 1997), no study has yet examined the statistical treatment of E-dependent changes in $\phi_{\mathrm{PSII}}$ and rETR. Given the increasing prevalence of active fluorescence measures in the literature (Suggett et al. 2011), such a review is warranted.

In this manuscript, $\operatorname{rETR}(\mathrm{E})$ and $\phi_{\mathrm{PSII}}(\mathrm{E})$ refer to E-dependent changes of rETR and $\phi_{\mathrm{PSI}}$ respectively. Methodologically, $\phi_{\text {PSII }}(\mathrm{E})$ is almost never directly modeled, with only two exceptions to the best of our knowledge (Laws et al. 2002; Smyth et al. 2004). Instead measures of $\phi_{\text {PSII }}$ are normally scaled to rETR $\left(\mathrm{rETR}=\phi_{\text {PSII }} \times \mathrm{E}\right)$ whose E-dependency is routinely modeled with published photosynthetic irradiance (PE) models that yield well understood physiological parameters such as lightlimited and light-saturated rETR ( $\alpha$ and $P_{M}$ respectively), and the irradiance $\left(\mathrm{E}_{\mathrm{K}}\right)$ that represents the inflection from $\alpha$ to $\mathrm{P}_{\mathrm{M}}$. This approach is statistically flawed as, unlike traditional PE measurements, the response variable (rETR) is explicitly dependent on the predictor variable (E). As $\phi_{\text {PSII }}$ is equivalent to rETR normalized to E, Laws et al. (2002) demonstrate that $\phi_{\text {PSII }}(\mathbf{E})$ can be modeled by normalizing a given PE model to $\mathrm{E}$, thus retaining PE model parameters (Smyth et al. 2004). An example is shown in Fig. 1 where $\operatorname{rETR}(\mathrm{E})$ and $\phi_{\mathrm{PSII}}(\mathrm{E})$ are modeled as an exponential function (Webb et al. 1974). 

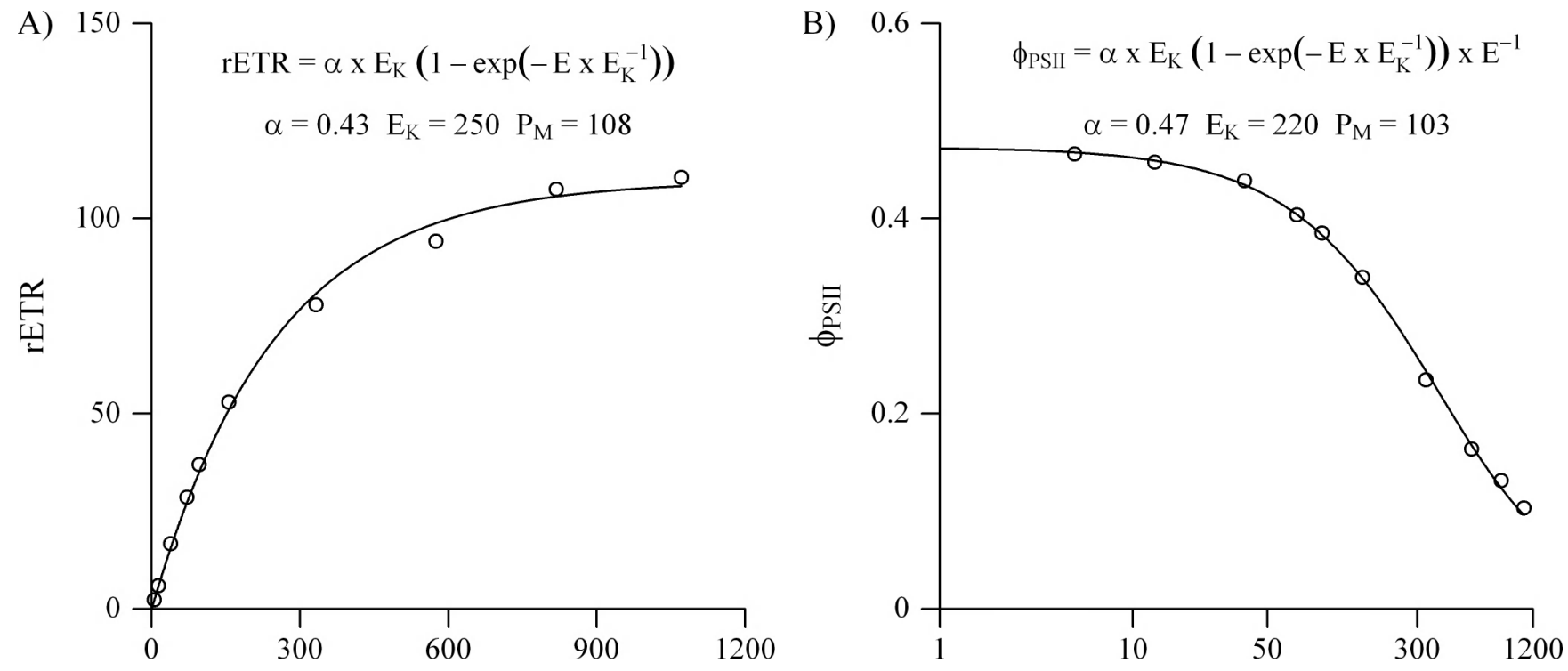

$\operatorname{PAR}\left(\mu \mathrm{mol} \mathrm{m} \mathrm{m}^{-2} \mathrm{~s}^{-1}\right)$

Fig. 1. An FLC dataset simultaneously modeled as A) $r E T R(E)$ and B) $\Phi P S I I(E)$ using Webb et al. (1974). The E axis in B) is log-transformed for clarity. PE parameters are shown and differ between modeling approaches.

In this article, we demonstrate that modeling FLC data as $\phi_{\text {PSII }}(\mathrm{E})$ is statistically more robust than modeling rETR(E). First, we demonstrate how the measurement error of rETR increases with $\mathrm{E}$ and document how this induces significant uncertainty in computed PE parameters. Next, a large FLC dataset is introduced and simultaneously fitted to four commonly cited PE models implemented in their original and E-normalized forms (Table 1). Four statistical criteria used to evaluate the efficacy of nonlinear models (Motulsky and Ransnas 1987) are presented for each model. PE parameters (presented in Table 1) are evaluated for their level of significance ( $P$ value) and relative standard error ( $R S E$, equal to the standard error normalized to parameter estimate). Model fits are evaluated for their root mean square value (RMS, equal to the square root of the sum of square residuals normalized to degrees of freedom in the regression), and the distribution of model residuals (observed - predicted) as they vary with $\mathrm{E}$.

\section{Materials and procedures}

\section{Study site and sampling}

Fluorescence light curve data were performed on natural phytoplankton communities across a seasonal and nutritional gradient in the western Wadden Sea and nearby Lake Ijsmeer, Netherlands. Water samples were collected every hour over a diurnal cycle at two discrete depths during four research cruises in February, March, April, and September 2010 at 6 different stations. Table 2 lists the mean and the range in water temperature, Chlorophyll $a$, dissolved inorganic nitrogen (DIN), soluble reactive phosphorus, and silicate concentrations along with the number of FLCs acquired during each cruise.

\section{Fluorescence light curve measurements}

Each FLC was measured with a FAST ${ }^{\text {act }}$ single turnover fluorometer (Chelsea Technologies Group), which exposes a water sample to a set of user-defined irradiance levels applied incrementally. Here phytoplankton were incrementally exposed to 10 irradiance levels (range 5 to $1071 \mu \mathrm{mol} \mathrm{m} \mathrm{m}^{-2} \mathrm{~s}^{-1}$ ) at 30 -s intervals. A temperature controlled water bath attached to the FAS$\mathrm{T}^{a c t}$ ensured that water sample remained at in situ temperatures. Fluorescence induction curves were user-defined and programmed to consist of $100 \mu$ s flashlets applied every $2 \mu \mathrm{s}$ followed by 20 relaxation flashlets applied every $49 \mu$ s (Kolber et al. 1998). Five induction curves spaced $100 \mathrm{~ms}$ apart were

Table 1. List of PE models used in this study to fit E-dependent changes in rETR and $\phi_{\mathrm{PSII}}$.

\begin{tabular}{|c|c|c|}
\hline Reference & rETR(E) & $\phi_{\text {PSII }}(\mathrm{E})$ \\
\hline Webb et al. 1974 & $\alpha \times E_{k} \times\left[1-e\left(-E \times E_{k}\right)\right]$ & $\alpha \times E_{k} \times\left[1-e\left(-E \times E_{k}\right)\right] \times E^{-1}$ \\
\hline Jassby and Platt 1976 & $\alpha \times \mathrm{E}_{\mathrm{k}} \times \tanh \left(\mathrm{E} \times \mathrm{E}_{\mathrm{K}}^{-1}\right)$ & $\alpha \times \mathrm{E}_{\mathrm{K}} \times \tanh \left(\mathrm{E} \times \mathrm{E}_{\mathrm{K}}^{-1}\right) \times \mathrm{E}^{-1}$ \\
\hline Platt et al. 1980 & $P_{s} \times\left[1-e\left(-\alpha \times E \times P_{s}^{-1}\right) \times e\left(-\beta \times E \times P_{s}^{-1}\right)\right]$ & $P_{S} \times\left[1-e\left(-\alpha \times E \times P_{S}^{-1}\right) \times e\left(-\beta \times E \times P_{s}^{-1}\right)\right] \times E^{-1}$ \\
\hline Eilers and Peeters $1988^{\dagger}$ & $E \times\left(a \times E^{2}+b \times E+c\right)^{-1}$ & $\left(a \times E^{2}+b \times E+c\right)^{-1}$ \\
\hline
\end{tabular}

${ }^{\dagger}$ For Eilers and Peeters (1988): $a=\left(\alpha \times E_{O p t}{ }^{2}\right)^{-1}, b=P_{M}{ }^{-1}-2 \times\left(\alpha \times E_{O p t}\right)^{-1}, c=\alpha^{-1}$. 
Table 2. List of cruises in 2010 and attendant water quality parameters. The range is given between brackets.

\begin{tabular}{lcccccc}
\hline $\begin{array}{l}\text { Cruise } \\
\text { dates }\end{array}$ & $\begin{array}{c}\text { Number } \\
\text { of FLCs }\end{array}$ & $\begin{array}{c}\text { Water temperature } \\
(\mathrm{Deg} \mathrm{C})\end{array}$ & $\begin{array}{c}\text { Chlorophyll a } \\
\left(\mathbf{m g ~ m}^{-3}\right)\end{array}$ & DIN $(\boldsymbol{\mu M})$ & $\begin{array}{c}\text { Soluble reactive } \\
\text { phosphorus }(\boldsymbol{\mu M})\end{array}$ & Silicate $(\boldsymbol{\mu M})$ \\
\hline 15-19 Feb & 75 & $0.5(0-1.6)$ & $7.0(2.5-27.2)$ & $85.5(50.5-179.3)$ & $0.70(0.19-0.86)$ & $42.9(24.0-94.3)$ \\
22-26 Mar & 129 & $6.6(5.2-12.1)$ & $27.1(2.5-91.6)$ & $51.2(24.3-194.7)$ & $0.04(0.004-0.20)$ & $2.3(0.1-25.3)$ \\
3-7 May & 119 & $10.7(7.8-12.1)$ & $14.3(5.7-36.6)$ & $31.0(2.2-158.1)$ & $0.39(0.05-1.84)$ & $5.2(0.3-14.1)$ \\
6-10 Sep & 89 & $15.6(13.6-16.5)$ & $30.3(9.6-131.7)$ & $11.2(0.3-21.5)$ & $0.19(0.04-0.40)$ & $14.9(0.3-76.0)$ \\
\hline
\end{tabular}

averaged by the Chelsea FastPro software into a single composite induction curve; this was repeated every $5 \mathrm{~s}$ allowing for approximately 6 composite curves per 30 -s irradiance level. The FastPro software models fluorescence $\left(F_{n}\right)$ as a function of the number of closed reaction centers $\left(\mathrm{C}_{\mathrm{n}}\right)$, where $\mathrm{n}$ is the excitation flashlet $(\mathrm{n}=1,2 \ldots 100)$, using the Kolber et al. (1998) model shown in Eqs. 1 and 2. This model derives four fluorescence parameters in actinic light $\left(\mathrm{F}^{\prime}, \mathrm{F}_{\mathrm{M}}^{\prime}, \sigma_{\mathrm{PSII}}{ }^{\prime}, \mathrm{p}\right)$, where $\mathrm{F}^{\prime}$ and $\mathrm{F}_{\mathrm{M}}{ }^{\prime}$ are the minimum and maximum fluorescence yield in actinic light, $\sigma_{\text {PSII }}{ }^{\prime}\left(\mathrm{nm}^{2}\right)$ is the effective absorption cross section in actinic light, and $\mathrm{p}$ is a dimensionless integer representing the degree of connectivity between photosystem II reaction centers. From these parameters $\phi_{P S I I}$ is derived as $\left(\mathrm{F}_{\mathrm{M}}{ }^{\prime}-\right.$ $\left.\mathrm{F}^{\prime}\right) / \mathrm{F}_{\mathrm{M}}$. At each irradiance, six measures of $\phi_{\mathrm{PSII}}$ were averaged into a single composite value. Background fluorescence was determined by measuring sample filtrate (filtered through a Whatman GF/F filter) in the FAST ${ }^{a c t}$ and subtracted from all fluorescence measurements following Suggett et al. (2006).

$$
\begin{gathered}
\mathrm{C}_{\mathrm{n}}=\mathrm{C}_{\mathrm{n}-1}+\sigma_{\text {PSII }}{ }^{\prime} \times\left(1-\mathrm{C}_{\mathrm{n}-1}\right) \times\left(1-\mathrm{p} \times \mathrm{C}_{\mathrm{n}-\mathrm{l}}\right)^{-1} \\
\mathrm{~F}_{\mathrm{n}}=\mathrm{F}^{\prime}+\left(\mathrm{F}_{\mathrm{M}}^{\prime}-\mathrm{F}^{\prime}\right) \times \mathrm{C}_{\mathrm{n}} \times(1-\mathrm{p}) \times\left(1-\mathrm{p} \times \mathrm{C}_{\mathrm{n}}\right)^{-1}
\end{gathered}
$$

The FastPro software does not quantify the standard errors of the various fluorescence parameters derived from fitting induction curves to the Kolber et al. (1998) model. Rather, the standard error that is given in the software refers to the standard error of the linear regression of the last 24 flashlets used to establish the initial value for $\mathrm{F}_{\mathrm{M}}{ }^{\prime}$ (Kevin Oxborough pers. comm.). To estimate the standard error of $\phi_{\mathrm{PSII}}$ across FLCs, induction curves $(n=4578)$ from a subset of FLCs $(\mathrm{n}=77)$ were fit to the Kolber et al. model (1998) using the open source statistical program R (R Development Core Team 2011).

\section{Statistical methods}

Nonlinear least squares regression of FLC data were performed using the 'modFit' function in the R package ' $\mathrm{FME}^{\prime}$ specifically designed for mathematical modeling of environmental data (Soetaert and Petzoldt 2010). The versatility of this function allows the user to select different numerical algorithms and easily extract relevant statistical parameters. In this study, numerical fitting of FLC data were performed using the Nelder-Mead algorithm. This algorithm was selected as it yielded more statistically significant model parameters (Table
1) relative to other algorithms tested (Levenberg-Marquardt, Gauss-Newton, and Port). A sample script written in the opensource statistical software $\mathrm{R}$ that invokes the statistical tests used here accompanies this manuscript as Web Appendix I.

\section{Assessment}

Measurement precision of PSII quantum efficiency and relative electron transport

Fig. 2 illustrates how measurement precision of $\phi_{\mathrm{PSII}}$ and rETR vary with $\mathrm{E}$. Panel A shows representative single turnover induction curves in the presence of low and high light (5 and $1071 \mu \mathrm{mol} \mathrm{m} \mathrm{m}^{-2} \mathrm{~s}^{-1}$ ). The high light induction curve is repeated in Panel $\mathrm{B}$ with the axis magnified. For each induction curve the statistical fit of $\mathrm{F}^{\prime}, \mathrm{F}_{\mathrm{M}}^{\prime}$, and $\phi_{\mathrm{PSII}}$ are stated \pm their respective standard error (SE) along with the root mean square (RMS), taken here as a proxy of induction curve noise. Panel $C$ presents boxplots of RMS values and the $S E$ of $\phi_{\text {PSII }}$ and rETR derived from a large set induction curves $(n=4578)$ across an E gradient. As RMS values show no E-dependency $(n=4578, P$ $>0.10$ ), the analysis in Fig. 2 demonstrates that induction curve noise is independent of E. However the relationship between $\mathrm{E}$ and the $S E$ of $\phi_{\text {PSII }}$ is biphasic. No significant $\mathrm{E}$ dependent changes in the $S E$ of $\phi_{\text {PSII }}$ occur below $150 \mu \mathrm{mol} \mathrm{m}^{-2}$ $\mathrm{s}^{-1}$, but above this irradiance, the $S E$ of $\phi_{\mathrm{PSII}}$ increases slightly but significantly with $\mathrm{E}(P<0.05)$. Examining $R M S$ values and $S E$ of $\phi_{\text {PSII }}$ together, we interpret the measurement precision of $\phi_{\text {PSII }}$ as being largely driven by the redox state of photosystem II. Below $150 \mu \mathrm{mol}$ photons $\mathrm{m}^{-2} \mathrm{~s}^{-1}$ most PSII reaction centers remain oxidized (inferred from $\mathrm{E}_{\mathrm{K}}$ measures below) and variable fluorescence $\left(\mathrm{F}_{\mathrm{M}}{ }^{\prime}-\mathrm{F}^{\prime}\right)$ is large relative to inherent noise. Above $150 \mu \mathrm{mol} \mathrm{m} \mathrm{m}^{-2} \mathrm{~s}^{-1}$ the progressive photochemical reduction of PSII reaction centers flattens induction curves $\left(\mathrm{F}_{\mathrm{M}}{ }^{\prime}\right.$ and $F^{\prime}$ converge) and variable fluorescence decreases relative to noise, thereby increasing the $S E$ of $\phi_{\mathrm{PSII}}$.

Fig. 2C also documents the $S E$ of rETR across an E gradient $(n=4578)$. The $S E$ of rETR is equal to the product of the $S E$ of $\phi_{\mathrm{PSII}}$ and its attendant $\mathrm{E}$ value (where we have assumed the $S E$ of $\mathrm{E}$ is constant). As $\mathrm{E}$ proportionality amplifies the $S E$ of $\phi_{\mathrm{PSI}}$ in this calculation, the $S E$ of rETR significantly increases with $\mathrm{E}(n=4578, P<0.05)$. The analysis presented in Fig. 2 is specific to single turnover fluorometers that allow error estimates to be derived from induction curves. Many FLCs are derived from multiple turnover fluorometers (e.g., PAM fluorometers) in which single measures of $\mathrm{F}_{\mathrm{M}}{ }^{\prime}$ and $\mathrm{F}^{\prime}$ preclude any statistical 


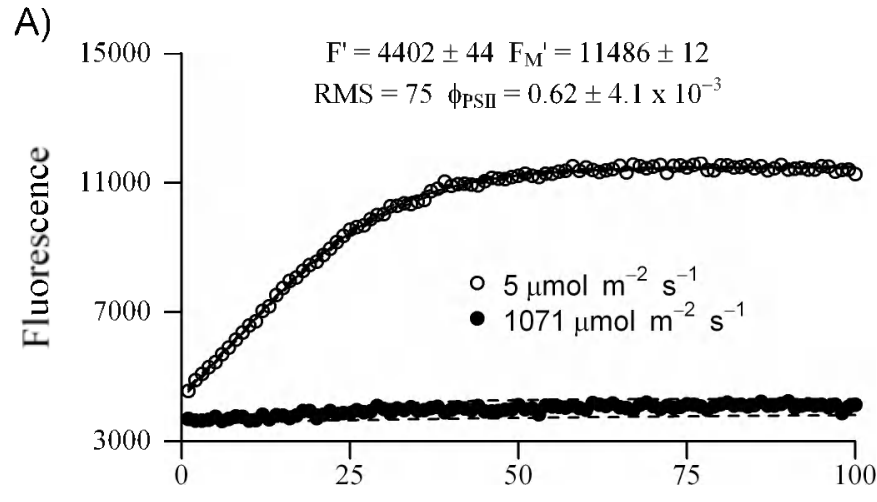

Flashlet

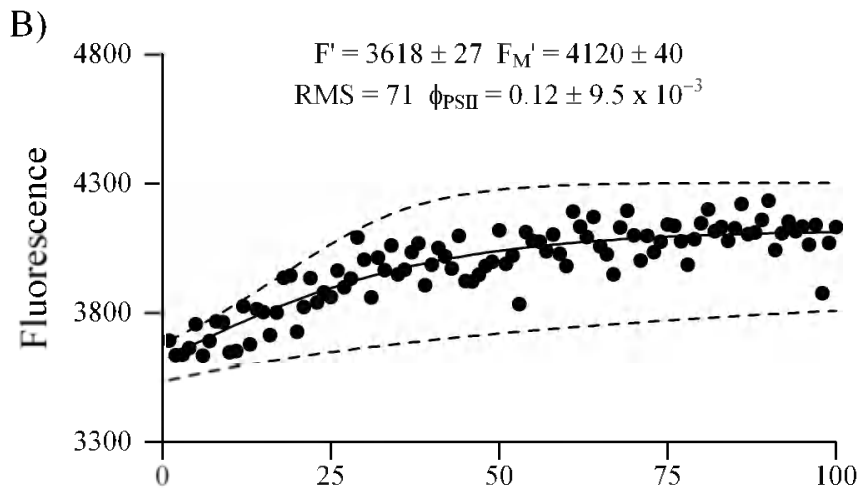

Flashlet

C)
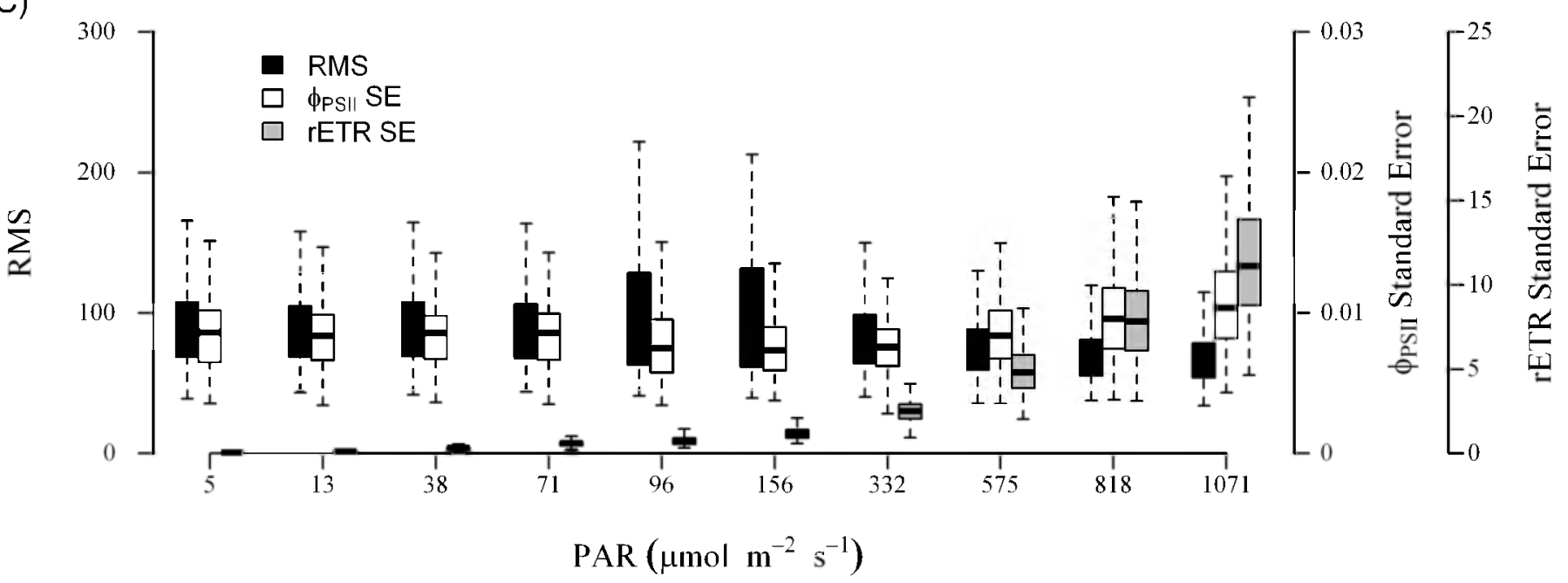

Fig. 2. A) Single turnover induction curves from a single sample with actinic irradiances of 5 and $1071 \mu \mathrm{mol}^{-2} \mathrm{~s}^{-1}$. The high light induction curve is shown again in B) with the axis magnified. The solid and dashed lines in each panel represent the Kolber et al. (1998) model fit and its $95 \%$ confidence intervals, though these are barely visible in Panel A. For each panel, the root mean square (RMS) of the fit is shown with resultant values of $F^{\prime}$, $F^{\prime} M$ and $\Phi P S I I \pm$ their respective standard error (SE). C) Boxplots of RMS values and the SE of $\Phi P S I I$ and rETR across an E gradient derived from a large set induction curves $(n=4578)$ from a subset of FLCs $(n=77)$. The upper and lower boundary of each box is the 75 th and 25 th percentile, whiskers represent the minimum and maximum values, and the solid line is the median.

error analysis. As fluorometers giving a multiple turnover flash measure $\mathrm{F}_{\mathrm{M}}$ ' with modulated light, such measures may have a higher signal to noise ratio at a given irradiance than single turnover fluorometers (Kromkamp and Forster 2003). But whatever active fluorometer is used, the convergence of $\mathrm{F}$ ' and $\mathrm{F}_{\mathrm{M}}{ }^{\prime}$ caused by reduction of PSII reaction centers makes measures of $\phi_{\mathrm{PSII}}$ prone to relatively large errors in high light, as experienced by most users of active fluorometers. Thus the measurement precision of FLC data runs counter to traditional PE assays (e.g., ${ }^{14} \mathrm{C}$ ) where the most precise measures are typically measured under high $\mathrm{E}$.

Fig. 3 illustrates the effects of rETR and $\phi_{\text {PSII }}$ measurement precision on PE parameters. Shown in Fig. 3A and 3B, a set of synthetic FLCs $(n=1000)$ were derived at ten E levels using the original and E-normalized PE model of Webb et al. (1974), respectively. At each E level, a random rETR or $\phi_{\mathrm{PSII}}$ value was generated from a normal distribution with a mean value derived from preset PE parameters $\left(\alpha=0.5, \mathrm{E}_{\mathrm{K}}=200\right.$, and $\mathrm{P}_{\mathrm{M}}=$ $100)$ and with a standard deviation equivalent to their respective standard error taken from Fig. 2C (and shown as vertical error bars in Fig. 3A and B). Each set of randomly generated rETR and $\phi_{\text {PSII }}$ measures were then refit to the model of Webb et al. (1974). Fig. 3C, D, and E show boxplots of the PE parameters derived from these fits. Mean PE parameters for $\operatorname{rETR}(\mathrm{E})$ and $\phi_{P S I I}(E)$ models were not statistically different than their original value $(t$-test, $P<0.05)$. However Fig. 3 clearly shows that, relative to the synthetic $\phi_{\text {PSI }}$ data, E-dependent increases in the $S E$ of rETR induce significantly larger variance in computed PE model parameters ( $F$-test, $P<0.05$ ).

\section{Model performance}

Table 3 summarizes statistical and model parameters derived by fitting the models listed in Table 1 to a large set of FLC measurements $(n=412)$. In this section, we ignore differences in the measurement precision of rETR and $\phi_{\text {PSII }}$ as 

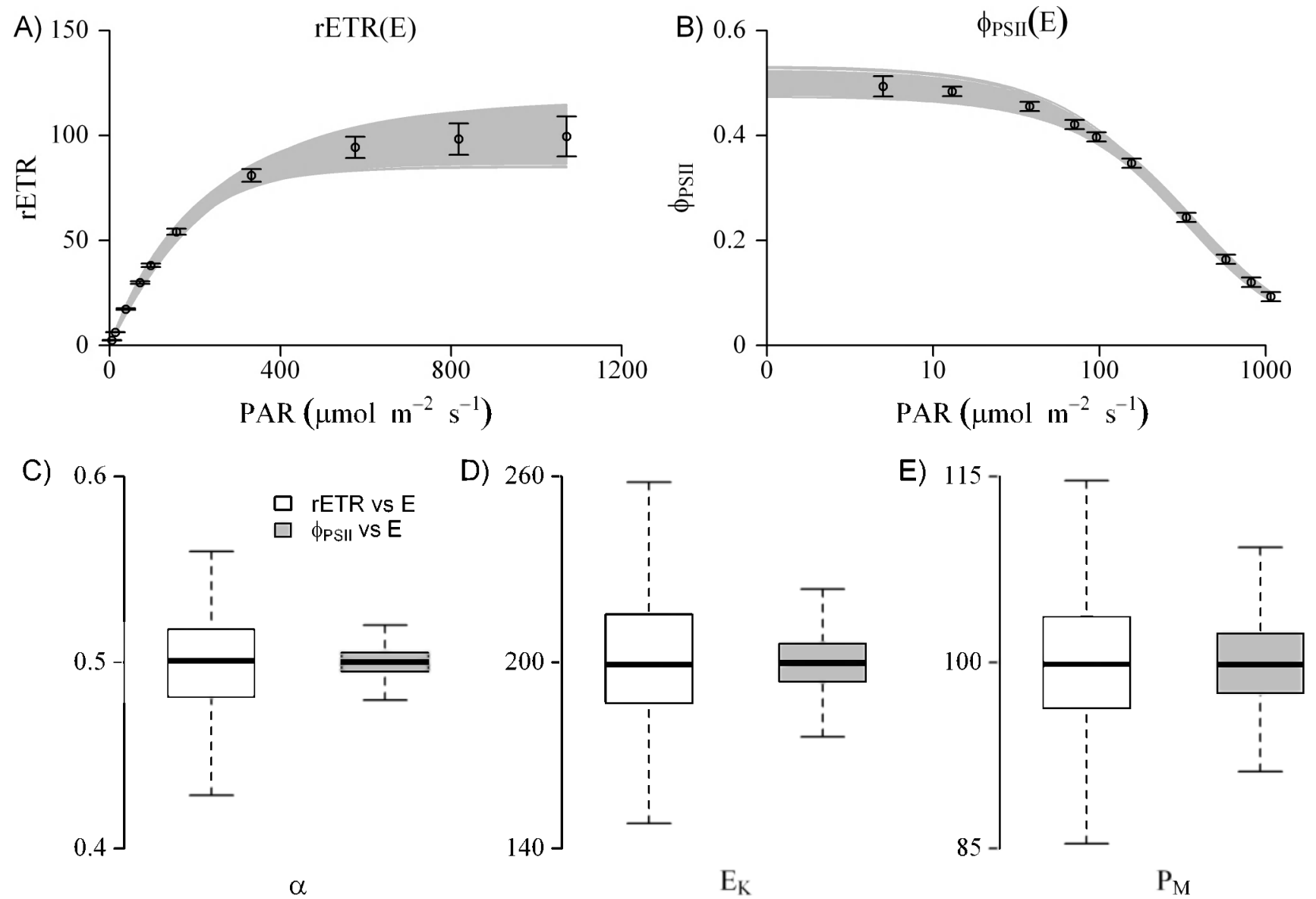

$\alpha$

$\mathrm{E}_{\mathrm{K}}$

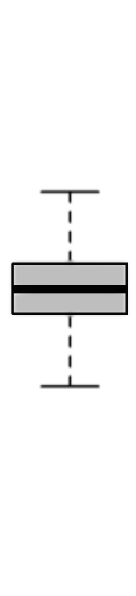

Fig. 3. Sensitivity analysis showing synthetic FLCs $(n=1000)$ derived from Webb et al. (1974) where $\alpha=0.5$ and EK $=200 \mu \mathrm{mol} \mathrm{m}^{-2} \mathrm{~s}^{-1}$, and the model is implemented in A) its original form and B) its E-normalized form. Grey lines are the synthetic curves generated from normal distributions with computed mean values shown as circles, and standard deviations equivalent to the respective standard errors of rETR(E) and $\Phi P S I I(E)$ taken from Fig. $2 C$ and shown here as vertical error bars. C-E) Boxplots, as described in Fig. 2, showing the distribution of computed PE parameters from the synthetic rETR(E) and $\Phi P S I I(E)$ curves.

Table 3. PE models applied to a FLC dataset $(n=412)$ in their original and E-normalized form. Next to each model $n$ states the number of FLC fits with statistically significant $(P<0.05)$ model parameters. Mean model parameters are given with their relative standard error $(R S E)$ in brackets. The mean model root mean square $(R M S)$ is computed only for FLCs that yielded statistically significant model parameters $(P<0.05)$. An ${ }^{*}$ denotes model parameters that are significantly different $(P<0.05)$ between rETR(E) and $\phi_{P S I I}(E)$ models.

\section{PE model}

Webb et al. 1974

rETR(E)

$\phi_{\mathrm{PSI}}(\mathrm{E})$

Jassby and Platt 1976

$\operatorname{rETR}(\mathrm{E})$

$\phi_{\mathrm{PSI|}}(\mathrm{E})$

Platt et al. 1980

$\operatorname{rETR}(\mathrm{E})$

$\phi_{\mathrm{PSII}}(\mathrm{E})$

Eilers and Peeters 1988

$\operatorname{rETR}(\mathrm{E})$

$\phi_{\text {PSII }}(\mathrm{E})$

395

409 $n$

412

412

412

412

167

77

PE Parameters (RSE)

Model RMS $\left(10^{-2}\right)$

$\alpha$

$0.47(5.2 \%)$

$0.47(1.3 \%)$

$\alpha$

$0.37(6.7 \%)^{*}$

$0.44(2.2 \%)^{*}$

$\alpha$

$0.49(2.4 \%)^{*}$

$0.48(0.7 \%)^{*}$

$\alpha$

$0.46(10.7 \%)^{\star}$

$0.47(1.6 \%)^{\circ}$
$\mathrm{E}_{\mathrm{K}}$

$294(7.2 \%)$

$270(5.0 \%)$

$\mathrm{E}_{\mathrm{K}}$

$335(8.9 \%)^{*}$

$233(8.3 \%)^{*}$

$\beta$

$2(8.5 \%)$

$0(4.0 \%)$

$\mathrm{E}_{\mathrm{OPT}}$

$918(16.7 \%)$

$815(14.7 \%)$
$P_{M}$

$132(17.2 \%) \quad 2.8$

$128(11.4 \%) \quad 1.0$

$P_{M}$

$123(26.0 \%)^{*} \quad 5.7$

$103(19.4 \%)^{*} \quad 2.0$

$P_{s}$

$99(3.5 \%)^{*} \quad 2.4$

$108(6.5 \%)^{*} \quad 1.0$

$P_{M}$

$122(2.8 \%)$

3.7

1.1 
such measures are rarely computed. Next to each model the number of FLCs that yielded statistically significant $(P<$ 0.05) PE parameters is stated. Only models that do not simulate photoinhibition (Webb et al. 1974; Jassby and Platt 1976) yielded statistically significant model parameters across all FLCs. The short duration in which phytoplankton are exposed to saturating light in the FLC protocol used here likely minimizes photoinhibitory processes relative to longer traditional photosynthetic assays. Mean coefficients of determination $\left(r^{2}\right)$, commonly used to test the efficacy of a given model, exceed 0.97 for all models (data not shown). Despite high $r^{2}$ values throughout, Table 3 underscores the validity of directly modeling $\phi_{\text {PSII }}(\mathrm{E})$. Across all PE models, fitting data as $\phi_{\text {PSII }}(\mathrm{E})$ yielded significantly smaller $R M S$ values than fitting data as rETR(E) (paired t test, $P<0.05$ ). Table 3 also demonstrates that normalizing a PE model to E also yields systematic differences in model parameters. In Table 3, an asterisk indicates that computed PE parameters were significantly different (Mann-Whitney test, $P<0.05$ ) when data for a given model is fit as rETR(E) or $\phi_{P S I}(E)$. In this dataset $\mathrm{E}_{\mathrm{K}}$ was the most sensitive model parameter to $\mathrm{E}$ normalization. Strikingly when the hyperbolic tangent model (Jassby and Platt 1976) was implemented to model rETR(E), the resultant mean $\mathrm{E}_{\mathrm{K}}$ was, on average, 1.42-fold higher then when modeled as $\phi_{\text {PSII }}(\mathrm{E})$. Fig. 4 presents boxplots of model residuals (observed - predicted) as they vary with E. To compare residuals between the original and E-normalized models listed in Table 3 , all residuals computed for rETR(E) models are normalized to E. Thus Fig. 4 explicitly examines each given model's ability to predict E-dependent changes in $\phi_{\text {PSII }}$. As in Table 3, only the residuals of FLCs that yielded statistically significant model parameters are shown. In Fig. 4, an asterisk above the $95 \%$ outlier indicates that, at a given $\mathrm{E}$, the absolute value of the residual mean exceeds the nominal $\phi_{\text {PSII }}$ $S E$ (taken here as 0.01 from Fig. $2 \mathrm{C}$, $t$ test or Wilcox test, $P<$ $0.05)$. Across all ten $\mathrm{E}$ levels, only the $\phi_{\mathrm{PSII}}(\mathrm{E})$ implemented models of Webb et al. (1974) and Platt et al. (1980) yielded mean $\phi_{\text {PSII }}$ residuals significantly smaller than the assumed measurement error. All other models indicate some Edependency in model residuals. Fig. 4 also helps visualize persistent differences in PE parameters listed in Table 3. For example, modeling rETR(E) with Jassby and Platt (1976) and Platt et al. (1980) yielded, on average, the smallest and largest estimates of $\alpha$, respectively. Accordingly, Fig. 4 demonstrates that for these two respective models $\phi_{\text {PSII }}$ residuals are, on average, greater and less than 0 in the presence of low light. Inspection of model residuals also helps reconcile disparate estimates of $\mathrm{E}_{\mathrm{K}}$. Fig. 4 clearly shows that the $\phi_{\text {PSII }}(\mathrm{E})$ and rETR(E) implementation of Jassby and Platt (1976) underestimates and overestimates $\phi_{\mathrm{PSI}}$ respectively across the range of typical $\mathrm{E}_{\mathrm{K}}$ values. Table 3 shows the mean $\mathrm{E}_{\mathrm{K}}$ values derived from the $\phi_{\mathrm{PSII}}(\mathrm{E})$ implemented version of Webb et al. (1974) fall between the two extremes of Jassby and Platt (1976), accordingly the residuals with this model are close to 0 across the range of typical $\mathrm{E}_{\mathrm{K}}$ values. Regardless of PE model, Fig. 4 further illustrates that modeling FLC data as $\phi_{\mathrm{PSII}}(\mathrm{E})$ yields smaller and less variable residuals than the traditional rETR(E) approach.

\section{Model selection}

Overall fitting $\phi_{\mathrm{PSII}}(\mathrm{E})$ with the model of Webb et al. (1974) is deemed the most appropriate model for this dataset. Only the models of Webb et al. (1974) and Jassby and Platt (1976) yielded statistically significant model parameters for all FLCs $(n=412)$. Of these two models, when $\phi_{\mathrm{PSII}}(\mathrm{E})$ is fit to Webb et al. (1974), the relative standard error of model parameters are the lowest, and $\phi_{\mathrm{PSII}}$ residuals did not exceed the measurement error. Moreover the mean model RMS was significantly smaller than any other model (paired $t$ test, $P<0.01$ ), with the exception of $\phi_{\text {PSII }}(\mathrm{E})$ fitted with Eilers and Peeters (1988). This is not to say that the exponential model should be universally implemented for FLC data, rather we propose these statistical tests should be considered when selecting an appropriate model. A sample script written in the open-source statistical software $\mathrm{R}$ that invokes the statistical tests used here accompanies this manuscript as Web Appendix I.

\section{Discussion}

Whether as a tool to examine photosynthetic physiology or compute rates of phytoplankton production, derivation of photosynthetic parameters $\left(\alpha, E_{K^{\prime}}, P_{M}\right)$ from measures of active fluorescence are becoming increasingly common (Suggett et al. 2011). However, the current widespread practice by which measures of rETR are modeled as a function of E, analogous to classic PE methodology, violates a key statistical requirement of nonlinear regression. This statistical violation can be avoided by normalizing a given PE model to $\mathrm{E}$ such that $\phi_{\mathrm{PSII}}(\mathrm{E})$ is modeled directly (Laws et al. 2002). The equivalency of $\phi_{\mathrm{PSII}}(\mathrm{E})$ models to traditional PE models has long been recognized (Kiefer and Mitchell 1983), yet infrequently implemented (Smyth et al. 2004). Indeed the accompanying software of Walz fluorometers (WinControl 3.0, Heinz Walz GmbH, Effeltrich GE) currently models FLC data as E-dependent changes in rETR. Here we have used a series of statistical tests that clearly demonstrate that modeling the E-dependency of $\phi_{\mathrm{PSI}}$ avoids unnecessary amplification of $\phi_{\mathrm{PSI}}$ errors and yields more accurate and precise PE parameters. This study definitively demonstrates that modeling $\phi_{\mathrm{PSII}}(\mathrm{E})$ directly should be the new norm when interpreting FLC data.

The statistical approach taken here can also be applied to traditional PE measures. For example, Jassby and Platt (1976) stated "...we frequently found that the final parameter estimates for $\alpha$ were far outside the range of published values and grossly inconsistent with estimates of $\alpha$ made by subjective inspection of the data taken at low light levels." Our implementation of their model is consistent with this observation. The statistical tests used in this study help identify systematic biases in PE parameters (Frenette et al. 1993). 

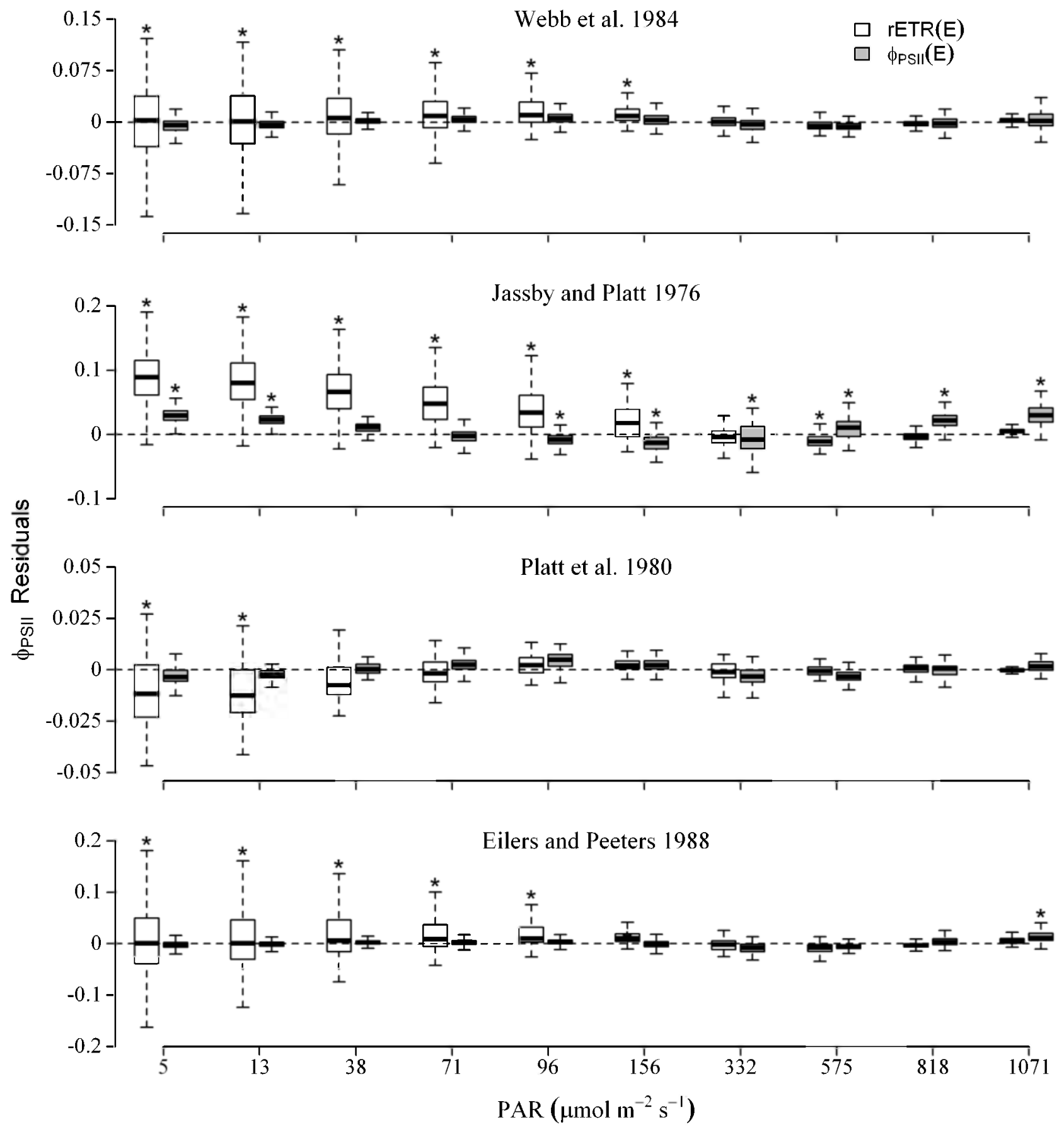

Fig. 4. Boxplots, as described in Fig. 2, showing the distribution of $\Phi P S I I$ residuals at 10 irradiance levels for the models and data listed in Table 3 . White and grey boxplots correspond to residuals derived from $\operatorname{rETR}(\mathrm{E})$ and $\Phi P S I I(E)$ models, respectively. An " above a given box indicates that the absolute value of the residual mean is significantly larger than $0.01(P<0.05)$. Note different $y$-axis scales between panels.

\section{References}

Aalderink, R. H., and R. Jovin. 1997. Estimation of the photosynthesis/irradiance $(\mathrm{P} / \mathrm{I})$ curve parameters from light and dark bottle experiments. J. Plankton Res. 19:1713-1742 [doi:10.1093/plankt/19.11.1713].
Eilers, P. H. C., and J. C. H. Peeters. 1988. A model for the relationship between light intensity and the rate of photosynthesis in phytoplankton. Ecol. Model. 42:199-215 [doi:10.1016/0304-3800(88)90057-9].

Frenette, J. J., S. Demers, L. Legendre, and J. Dodson. 1993. Lake of agreement among models for estimating the pho- 
tosynthetic parameters. Limnol. Oceanogr. 38(3):679-687 [doi:10.4319/lo.1993.38.3.0679].

Jassby, A. D., and T. Platt. 1976. Mathematical formulation of the relationship between photosynthesis and light for phytoplankton. Limnol. Oceanogr. 21:540-547 [doi:10.4319/ lo.1976.21.4.0540].

Kiefer, D. A., and B. G. Mitchell. 1983. A simple, steady state description of phytoplankton growth based on absorption cross-section and quantum efficiency. Limnol. Oceanogr. 28:770-776 [doi:10.4319/lo.1983.28.4.0770].

Kolber, Z. S., O. Prášil, and P. G. Falkowski. 1998. Measurements of variable chlorophyll fluorescence using fast repetition rate techniques: defining methodology and experimental protocols. Biochim. Biophys. Acta 1367:88106 [doi:10.1016/S0005-2728(98)00135-2].

Kromkamp, J. C. and R. M. Forster. 2003. The use of variable fluorescence measurements in aquatic ecosystems: Differences between multiple and single turnover measuring protocols and suggested terminology. Eur. J. Phycol. 38:103112 [doi:10.1080/0967026031000094094].

Laws, E., and others. 2002. Photosynthesis and primary productivity in marine ecosystems: Practical aspects and application of techniques. JGOFS Report No. 36.

Motulsky, H. J., and L. A. Ransnas. 1987. Fitting curves to data using nonlinear regression: a practical and non mathematical review. FASEB J. 1:365-374.

Platt, T., C. L. Gallegos, and W. G. Harrison. 1980. Photoinhibition and photosynthesis in natural assemblages of marine phytoplankton. J. Mar. Res. 38:687-701.
R Development Core Team. (2011). R: A language and environment for statistical computing. R Foundation for Statistical Computing. <http://www.R-project.org/>.

Soetaert, K., and T. Petzoldt. 2010. Inverse modeling, sensitivity and Monte Carlo analysis in $\mathrm{R}$ using package FME. J. Stat. Softw. 33:1-28.

Smyth, T. J., K. L. Pemberton, J. Aiken, and R. J. Geider. 2004. A methodology to determine primary production and phytoplankton photosynthetic parameters from Fast Repetition Rate Fluorometry. J. Plankton Res. 26:1337-1350 [doi:10.1093/plankt/fbh124].

Suggett, D. J., S. C. Maberly, and R. J. Maberly. 2006. Gross photosynthesis and lake community metabolism during the spring phytoplankton bloom. Limnol. Oceanogr. 51:3064-2076 [doi:10.4319/1o.2006.51.5.2064].

, O. Prasil, and M. A. Borowitzka. 2011. Chlorophyll fluorescence in aquatic sciences: Methods and applications. Springer.

Webb, W. L., M. Newton, and D. Starr. 1974. Carbon dioxide exchange of Alnus rubra: A mathematical model. Oecologia 17:281-291 [doi:10.1007/BF00345747].

White, A. J., and C. Critchley. 1999. Rapid light curves: A new fluorescence method to assess the state of the photosynthetic apparatus. Photosynth. Res. 59:63-72 [doi:10.1023/ A:1006188004189].
Submitted 15 December 2011 Revised 22 May 2012 Accepted 6 June 2012 